\title{
STARBASE: A Network of Fully Autonomous Telescopes for Hands-on Science Education
}

\author{
Richard Gelderman, David Barnaby, Michael Carini, Karen Hackney, \\ Richard Hackney, Charles McGruder, and Roger Scott \\ Western Kentucky University, Dept. of Physics and Astronomy, \\ Bowling Green, Kentucky, USA 42101
}

\begin{abstract}
Students Training for Achievement in Research Based on Analytical Space-science Experiences (STARBASE) is being established to provide exciting hands-on research opportunities for students. STARBASE is a network of networks, consisting of dedicated hardware, universities, professional astronomers, teachers, and students all working together in scientific investigations. Funded through the NASA Office of Space Science, the STARBASE network is working to bring major science research projects to motivated students all over the globe.
\end{abstract}

Western Kentucky University is the lead institution for STARBASE Students Training for Achievement in Research Based on Analytical Spacescience Experiences. In 1999, WKU received funding from NASA's Office of Space Science, allowing us to begin to put into place the networks of hardware, universities, teachers, and students which comprise STARBASE.

The Network of Hardware: Fully autonomous observatories have recently been made possible through the revolutionary advances in computing technology. Advances in computing power and networking allow even remote institutions with limited budgets to operate a small telescope at a world-class observing site. The STARBASE network of observatories - with the first of three fully autonomous meter-class telescopes coming on-line - is another example of the recent efforts to establish global networks of small telescopes.

The 0.6-meter Western Kentucky University telescope is located near Bowling Green, Kentucky (latitude $=+36^{\circ} 55$, longitude $=+86^{\circ} 36.7$, elevation $225 \mathrm{~m}$ ) . The telescope system has been refurbished and automated by Astronomical Consultants and Equipment, Inc. of Tucson, Arizona. Commissioning tests were being performed at the time of this conference, with the intention of achieving robotic operation in the spring of 2001.

The 1.3-meter Robotically Controlled Telescope (RCT) is located at the Kitt Peak National Observatory (latitude $=+31^{\circ} 57$, longitude $=+111^{\circ} 35.7$, elevation $2064 \mathrm{~m}$ ). The refurbishment and automation of the former KPNO 1.3meter is being undertaken by EOS Technologies, Inc. of Tucson, Arizona, and recommissioning of the RCT is scheduled for early 2002.

Negotiations to locate a new meter-class fully autonomous telescope at the Wise Observatory in Israel (latitude $=+30^{\circ} 36$, longitude $=-31^{\circ} 45.8$, elevation $900 \mathrm{~m}$ ) are progressing well, though no start date has been established.

An additional observatory at a site in eastern Asia is desired, but funding had not yet been secured as of this conference. 
Other hardware requirements include the need for a powerful computer network to archive and distribute the data. Again, the remarkable advances in computing technology make STARBASE possible - allowing storage of terrabytes of data and daily transfers to the end users of gigabytes of data (both of which were only recently unthinkable volumes). We are relying on a combination of Unix, Linux, and WinNT machines to host the hard disks, tapes, and CDRs which comprise our data retrieval system.

The Network of Universities: The research programs which are the foundation of STARBASE include: photometric detection of extrasolar planets transiting their parent star (Everett \& Howell 2001); photometric monitoring of quasars and blazars (Clements \& Carini 2001); and emission line imaging of AGN, starburst galaxies, and Galactic nebulosities (Buckalew et al. 2000). Shared research interests and a joint commitment to basic science education bind our partners in a common purpose. The professional astronomers in the STARBASE network of universities firmly believe that the education of both secondary and college students is best served by directly involving them in scientific investigation.

The Network of Teachers and Students: Our educational plans address the need for hands-on, inquiry-based learning in order for students to develop the ability to think and work as scientists. When all the networks are operating together, the standardized hardware and software will enable the teachers and students in the STARBASE network to develop meaningful research projects and to collect, reduce, and analyze their own data in order to carry out their projects. There will be no 'canned' STARBASE investigations, each group of students will have a unique and original experience as they plan, execute, and present the results of their own scientific projects.

The STARBASE network of teachers and students is initially being recruited from rural school districts in the southeast United States: Kentucky, South Carolina, Tennessee, and West Virginia. Training workshops are planned for the Kentucky and Arizona telescopes to introduce teachers to observational astronomy and the procedures required to establish a student-originated investigation or to participate in existing STARBASE research programs. In addition, we are developing web-based 'smart software' to assists students in formulating successful observing plans, as well as to provide instruction in observational astronomy as each student realizes the need for that information.

STARBASE students are already assuming responsible roles in the development of facilities, and will eventually be involved in all phases of the project. They are working with modern mechanical, electronic, and computer systems in support of the hardware development, and presenting the results of their work at local, regional, and national conferences.

\section{References}

Buckalew, B., Dufour, R.J., Shopbell, P., and Walter, D.K. 2000, AJ, 120, 2402 Clements, S.D. and Carini, M.T. 2001, AJ, 121, 90

Everett, M.E. and Howell, S.B. 2001, PASP, submitted 